\title{
ON ELECTRODYNAMICAL SELF-INTERACTION
}

\author{
JERZY KIJOWSKI \\ Centre for Theoretical Physics, Polish Academy of Sciences \\ Al. Lotników 32/46, 02-668 Warszawa, Poland
}

\section{Dedicated to Prof. Iwo Biatynicki-Birula on the occasion of his 60th birthday}

It is shown that the classical Maxwell theory admits a simple and natural renormalization of the self-interaction energy. This leads to a consistent, local and causal, relativistic theory of the Maxwell field interacting with classical, charged, point-like particles. The theory may be regarded as a simple and necessary completion of special relativity. The renormalization method proposed here is a realization of Einstein's idea of "deriving equations of motion from field equations". It is shown that Dirac's "3-dots" equation does not describe a fundamental law of physics, but only a specific family of solutions of our theory, corresponding to a specific choice of the field initial data.

PACS numbers: 03.50.De, 04.20.Me

\section{Introduction}

Usual approaches to the problem of interaction between electromagnetic field and classical point-like particles lead to infinite self-interaction energy. This way classical electrodynamics splits into two incompatible parts: (1) Maxwell theory for the evolution of the electromagnetic field with given sources and (2) Lorentz theory of motion of test particles (i.e. point particles, which themselves do not influence the field). Any attempt to describe the composed "particles + field" system by putting together these two parts leads to a contradiction: due to Maxwell equations the Lorentz force will always be infinite.

There have been many attempts to "renormalize" this infinity (e.g. [1]), based on different "cut-offs", but none of them is satisfactory. Most of these theories assume finite particle's dimension. But then, an infinite amount of information (Poincaré stresses) is necessary to describe the interior of the particle. Many efforts have been made to replace this infinite number of internal degrees of freedom by a finite number of "collective degrees of freedom" of mechanical nature, i.e. to approximate field theory by mechanics. The easiest way to do this consists in assuming that the particle is rigid (see [3] and [4]), but then the theory is no longer relativistic. Moreover, the results obtained this way are highly dependent upon the 
dimension of the particle. Its radius $r_{0}$ plays the role of a "cut-off parameter" and the resulting "renormalized Lorentz force" tends again to infinity when $r_{0} \rightarrow 0$.

A similar "cut-off" is provided by the non-linearity parameter in the Born-Infeld theory [5]. Again, the finite results of such a theory are unstable with respect to small changes of this parameter.

Another approach was proposed by Dirac (see [2]), who tried to eliminate the field from the composed "particles + field" system, and to calculate only the field's global influence on the particles' motion. Although some solutions of the Abraham-Lorentz-Dirac equation obtained this way, seem to describe well the "radiative friction" in specific physical situations, the theory is obviously inconsistent, because it breaks the symmetry of electrodynamics with respect to time reversal and admits non-physical, self-accelerating solutions. This is due to the fact that Dirac's approach is based on a non-physical decomposition of the field into the "retarded" and the "radiative" component and does not describe the interaction of particles and fields in terms of locally defined quantities. (It is interesting to note that the "good" solutions of the Abraham-Lorentz-Dirac equations are also solutions of the theory presented in the present paper - see Sec. 7.)

In this paper we present a consistent theory of the Maxwell electromagnetic field and point-like particles interacting with the field. Each particle is characterized by its rest mass $m$ and its electric charge $e$. Within this framework there is no possibility of describing multipole particles and the reason is not technical but fundamental, related with the $U(1)$-gauge structure of electrodynamics. The mass $m$ is "already renormalized" and represents the total energy (including the energy of the electromagnetic field surrounding the particle) of the particle at rest.

Physically, the theory is derived from an extended-particle model by a suitable approximation procedure, developed in Ref. [6]. Instead of looking for a trick enabling us to "renormalize" the infinite energy of the Coulomb field and make it finite, we observe that the integral representing the difference between the field energy carried by two solutions of Maxwell equations, corresponding to two trajectories which are tangent at the time $t_{0}$ is finite! Moreover, the same is true not only for the energy but for the total four-momentum (see Sec. 2). This observation leads immediately to an "already renormalized" formula for the total four-momentum of the system composed of particles and fields. Indeed, at any point $x(t)$ of the particle's trajectory we may define the total four-momentum of the system as the sum of two terms: (1) the total four-momentum of the uniformly moving particle (and its surrounding field) having at $x(t)$ the same velocity as our particle and (2) the difference between the two. The latter being already well defined and finite, it is only necessary to define the first one. Here, the extended particle model leads unambiguously to the value $m u^{\mu}$, where $u^{\mu}$ is the four-velocity of the particle.

This way, for any solution of Maxwell equations with a $\delta$-like sources, we assign the total four-momentum of the composed (particle + field) system to each point of the particle's trajectory. For a generic trajectory and a generic solution of Maxwell equations, the quantity obtained this way is not constant along the trajectory. We impose the conservation of the above total four-momentum as an additional condition which we add to the standard Maxwell theory. Together with this condition, the theory becomes finite and causal: the initial data for both 
particles and fields uniquely determine the future and the past of the system, although the Lorentz force acting on the particles remains always ill defined.

The total four-momentum being defined by an integral, its conservation is a non-local condition. Nevertheless, our theory is perfectly local. Indeed, we prove that, due to Maxwell equations, our conservation condition is equivalent to a boundary condition for the behaviour of the electromagnetic field on the particle trajectory. We call this condition the fundamental equation of the theory (see Sec. 4). The field equations of our theory are therefore precisely the linear Maxwell equations for the electromagnetic field with point-like sources. The new element which makes the system behave in a causal way is the above boundary condition, with the particle trajectory playing the role of the moving boundary.

Mathematically, the theory is therefore formulated in terms of a problem with moving boundary.

An important ingredient of our theory, which makes it work, is the following observation, presented in Sec. 3, concerning the mathematical structure of linear electrodynamics with $\delta$-like sources. For any solution of Maxwell equations which is regular outside of the sources, the particle's acceleration is uniquely given by the $\left(r^{-1}\right)$-term of the field in the vicinity of the particle. We stress that this is a property of Maxwell equations. Hence, the acceleration of the particle is implied by the field initial data, without assuming any equation of motion.

The above statement may look as a paradox: we have been always taught that, for a given trajectory, the field initial data for the unhomogeneous Maxwell equation may be chosen almost at will, the only constraint being that the divergence of the electric induction must match the ( $\delta$-like) electric charge density. What then happens if we change the $\left(r^{-1}\right)$-term of field initial data at $t=t_{0}$ and solve the Maxwell equation with the new data, keeping the same trajectory? This way we will obtain a solution for which the previous relation between the field and the acceleration is no longer valid!

The answer is very simple: the solution obtained this way is not regular. Its singularity propagates along the light cone from the point at which the relation between the $\left(r^{-1}\right)$-term of the field and the acceleration has been broken. The regularity condition really excludes such a behaviour.

In Sec. 4 we show also that the limit of our theory for $e \rightarrow 0$ and $m \rightarrow 0$ with their ratio being fixed, coincides with the Maxwell-Lorentz theory of test particles. However, for any finite $e$ the acceleration of the particle cannot be equal to the Lorentz force, the latter being always ill defined.

In Sec. 5 we sketch the proof of the fundamental equation. For this purpose we rewrite the field dynamics in the rest frame of the particle. This is an accelerated reference system and not an inertial one. Its use simplifies considerably all the subsequent calculations.

The complete many-particles case is discussed in Sec. 6. In particular, assuming that one particle is very heavy and practically does not move, we may consider its own Coulomb field as an "external field". This way we are led to an extension of our theory, where the light particle interacts not only with its own "radiation field", but also with an "external field" or simply a field produced by a heavy external device. This extended theory is also discussed in Sec. 6. 
Although the field equations of the theory are linear, the moving boundary condition makes it relatively difficult. The method developed in Sec. 5 enables us to replace the problem with moving boundary by a problem with fixed boundary, but the price we pay for it is an effective non-linearity of the resulting dynamical system. It is therefore highly non-trivial to produce exact solutions. There is, however, a one-parameter family of third order ordinary differential equations for the particle trajectory, which result from our theory and which may be useful to construct exact solutions in special physical situations. Each of them is obtained from a specific "Ansatz" for the field surrounding the particle. We analyze this method in Sec. 7 and show that, in particular, the Abraham-Lorentz-Dirac equation may be obtained this way as a particular example. Therefore, all the solutions of the Abraham-Lorentz-Dirac theory (also the self-accelerating ones) fulfill the dynamical equations of our theory. However, to maintain such a self-acceleration, a non-physical ("fine tuned") choice of field initial data is necessary, with an infinite amount of field energy prepared far away from the particle. In this way a curious role of Dirac's "time arrow" has been explained: it is not a fundamental property of the theory, but has been introduced by the choice of initial data. The theory itself remains symmetric with respect to time reversal and the Abraham-Lorentz-Dirac "force of radiative friction" may be changed completely (e.g. may be multiplied by -1 ) if we change field initial data.

Although the problems with moving boundaries are always technically more complicated, the conceptual structure of our theory is extremely simple. The author wonders why it was not discovered at least 70 years ago. The only reason for that could be the usual fear of theoretical physicists to deal with boundary problems (see [7]). Even when describing a particle in a finite box, people usually prefer to consider "periodic boundary conditions" than to use any realistic description of the boundary data for the field. Similarly, the ordinary description of the Hamiltonian structure of field theory is based on integration by parts and the dogma of vanishing of all the surface integrals obtained this way (cf. e.g. [8]). As we shall see, in electrodynamics of moving particles the surface integrals do not vanish. On the contrary, they carry the entire useful information about particle dynamics (see also [9-11]).

\section{Renormalized total four-momentum}

Here we define the total four-momentum $p_{\lambda}$ of the system composed of the point-like particle and surrounding Maxwell field. Let $y=q(t)$ or $y^{k}=q^{k}(t)$, $k=1,2,3$ with $t=y^{0}$, be the coordinate description of a time-like world line $\zeta$ with respect to a laboratory reference frame, i.e. to a system $\left(y^{\lambda}\right), \lambda=0,1,2,3$, of Lorentzian space-time coordinates.

We assume that the field $f_{\mu \nu}$ satisfies the standard vacuum Maxwell equations with point-like sources

$$
\partial_{[\lambda} f_{\mu \nu]}=0, \quad \partial_{\mu} f^{\nu \mu}=e u^{\nu} \delta_{\zeta},
$$

where $\delta_{\zeta}$ denotes the $\delta$-distribution concentrated on a smooth world line $\zeta$

$$
\delta_{\zeta}\left(y^{0}, y^{k}\right)=\sqrt{1-\left(v\left(y^{0}\right)\right)^{2}} \delta^{(3)}\left(y^{k}-q^{k}\left(y^{0}\right)\right) .
$$


Here $v=\left(v^{k}\right)$, with $v^{k}:=\dot{q}^{k}$, is the corresponding 3-velocity and $v^{2}$ denotes the square of its 3-dimensional length (we use the Heaviside-Lorentz system of units with $c=1$ ).

The field carries the Maxwell energy-momentum tensor

$$
T^{\mu}{ }_{\nu}=f^{\mu \lambda} f_{\nu \lambda}-\frac{1}{4} \delta_{\nu}^{\mu} f^{\kappa \lambda} f_{\kappa \lambda} \text {. }
$$

The tensor has an $r^{-4}$ singularity on the particle trajectory $\zeta$, corresponding to the $r^{-2}$ singularity of the field $f_{\mu \nu}$ and cannot, therefore, be integrated over 3-dimensional space-like hypersurfaces.

Take now two trajectories $\zeta_{1}$ and $\zeta_{2}$, which are tangent at a point $\left(t, q_{(1)}(t)\right)=$ $\left(t, q_{(2)}(t)\right)$. Let $f^{(1)}$ and $f^{(2)}$ be any solutions of the corresponding Maxwell equations (1). The following relative four-momentum, representing the difference between the four-momentum carried respectively by $f^{(1)}$ and $f^{(2)}$, is well defined and finite

$$
p_{\lambda}^{\mathrm{rel}}:=\mathrm{P} \int_{\Sigma}\left(T_{\lambda}^{\mu}\left(f^{(1)}\right)-T_{\lambda}^{\mu}\left(f^{(2)}\right)\right) n_{\mu} \mathrm{d} \Sigma
$$

where $\Sigma$ is any space-like hypersurface intersecting both trajectories at the point where they are tangent to each other. By $\mathrm{d} \Sigma$ we denote the 3-dimensional volume element on $\Sigma$ and "P" denotes the principal value of the singular integral, defined by removing from $\Sigma$ a sphere $K(0, r)$ around the particle and then passing to the limit $r \rightarrow 0$. We assume that $\Sigma$ fulfills standard asymptotic conditions at infinity (see e.g. [12]) killing all the surface integrals at infinity which arise in deriving energy-momentum conservation laws.

The quantity (4) is well defined because the $r^{-4}$ singularities of the two tensors cancel. Moreover, the $r^{-3}$ singularities are always odd (see [6] for more detailed analysis). This means that the values of the $r^{-3}$-term at the antipodes have opposite signs. IIence, their contribution will be canceled by the principal value procedure.

It can be proved (see again [6]) that the above quantity does not depend upon the choice of $\Sigma$ within the class of surfaces which intersect our trajectories always at the same point. It is relatively easy to prove this invariance within a class of hypersurfaces tangent to each other at the intersection with the trajectories. Indeed, due to Maxwell equations both tensors are conserved outside of the particle

$$
\nabla_{\mu} T_{\lambda}^{\mu}\left(f^{(1)}\right)=0=\nabla_{\mu} T_{\lambda}^{\mu}\left(f^{(2)}\right) \text {. }
$$

The difference between the values of the integral corresponding to two different $\Sigma$ 's is therefore equal to a boundary (surface) integral. The boundary is composed of two parts: infinity and a piece $\mathcal{V}$ of a tiny world tube of radius $r$ around the particle. The integral at infinity vanishes if the field fulfills standard asymptotic conditions at infinity (see again [12]). The 3 -dimensional volume of $\mathcal{V}$ behaves at least like $r^{4}$ (2-dimensional volume of the spheres behaves like $r^{2}$ and the 3-rd dimension of $\mathcal{V}$, equal to the time interval between different $\Sigma$ 's, behaves also like $r^{2}$ since they are tangent to each other at $r=0$ ). Hence, the integral over $\mathcal{V}$ of a function behaving like $r^{-3}$ vanishes in the limit $r \rightarrow 0$.

In Ref. [6] we give a more detailed analysis of the behaviour of the energy-momentum tensor in the vicinity of $\zeta$, which enables us to extend the above proof to the case of general $\Sigma$ 's, not necessarily tangent to each other. 
Formula (4) suggests, that for a given trajectory $\zeta$ and a given point $(t, q(t)) \in \zeta$ the total four-momentum of the "particle + field" system may be defined as the sum of two terms: (1) the total four-momentum of the uniformly moving particle (and its surrounding field), matching the velocity of our particle at $(t, q(t))$ and (2) their relative four-momentum. Hence, we define the total energy-momentum by the following formula:

$$
p_{\lambda}(t):=m u_{\lambda}(t)+P \int_{\Sigma}\left(T_{\lambda}^{\mu}-T_{(t)}{ }_{\lambda}\right) n_{\mu} \mathrm{d} \Sigma,
$$

where $\Sigma$ is any hypersurface which intersects the trajectory at the point $(t, q(t))$. By $T_{(t)}^{\mu}$, we denote the energy-momentum tensor of the Coulomb field, boosted in such a way that the position and the velocity of its singularity at $(t, q(t))$ match the corresponding position and the velocity of our particle. Due to the invariance of the above integral with respect to changes of $\Sigma$, the total four-momentum defined this way depends only on the point of the trajectory.

Formula (6) may also be derived from an extended particle model via the following approximation procedure.

We imagine our "particle at rest" as a stable, soliton-like solution of a hypothetical fundamental theory ("super theory") of interacting electromagnetic and matter fields. We assume this theory to be relativistic and gauge invariant. Moreover, we assume that for weak electromagnetic fields and vanishing matter fields (i.e. far away from the particles) the theory coincides with Maxwell electrodynamics. The parameters $e$ and $m$ are the total charge and the total mass (including the electromagnetic part) of the non-perturbed soliton. In particular, the parameter $m$ is an already renormalized mass, (or dressed mass), including the energy of the field surrounding the particle. Within this framework questions like "how big the bare mass of the particle is and which part of the mass is provided by the purely electromagnetic energy?" are meaningless. In the strong field region (i.e. inside the particles) the energy density may be highly non-linear and there is probably no way to divide it consistently into such two components.

Due to the relativistic invariance of the theory, there is a 6-parameter family of the "uniformly moving particle" solutions obtained from our soliton via Poincaré transformations.

A general moving particle is understood as a "perturbed soliton". This means that it is again an exact solution of the same "super theory", with its strong-field-region concentrated in the vicinity of a time-like world line $\zeta$, which is no longer a straight line, as it was for "uniformly moving particles".

Calculate the total four-momentum of such a solution. For this purpose we choose any $\Sigma$ and integrate the total energy-momentum tensor of the "super theory" over $\Sigma$. This integral may be decomposed as a sum of the total four-momentum of the "uniformly moving particle" solution and the corresponding difference. This way we obtain the formula analogous to (6), where the energy-momentum tensors under the integral sign correspond now to two different solutions of the "super theory". Outside of the particles they reduce to the Maxwell energy-momentum tensors, because the entire theory reduces to the Maxwell theory. 'The contribution from the strong field region (i.e. from the possibly strong matter fields) is negligible, because both solutions do not differ too much inside 
of the particles (this is implied by the stability of our soliton). This means that the purely electromagnetic contribution which we left in formula (6) is a good approximation of the real value.

Noether theorem applied to our "super theory" implies the conservation of the total four-momentum. But for a generic trajectory $\zeta$ and a generic solution of Maxwell equations (1) the quantity (6) is not conserved, i.e. it depends upon $t$. We conclude that only those solutions of Maxwell equations, for which $p_{\lambda}(t)$ does not depend on time, may approximate the real motion of the true "perturbed soliton", governed by the field equations of the "super theory".

We shall see in Sec. 5 that only three among these four equations are independent. As independent conditions we may use e.g. the three-momentum conservation law

$$
\frac{\mathrm{d}}{\mathrm{d} t} p_{j}(t)=0,
$$

which implies also the energy conservation

$$
\frac{\mathrm{d}}{\mathrm{d} t} p^{0}(t)=0 \text {. }
$$

The above equations, together with the Maxwell equations for the electromagnetic field, define a mathematically consistent, fully deterministic theory. Starting from an appropriate variational principle, it may be proved that $p_{k}(t)$ is really a momentum canonically conjugate to the particle position $q^{k}(t)$ and $p^{0}(t)=-p_{0}(t)$ (we take the signature $(-,+,+,+)$ of the space-time metric) is the Hamiltonian of the entire system composed of particles and fields.

\section{Boundary conditions for the Maxwell field}

Given a regular solution of (1), there is one-to-one correspondence between the $r^{-1}$ term of the field in the vicinity of each point $(t, q(t)) \in \zeta$ and the acceleration of the particle at this point. The formula - although relativistic invariant is relatively complicated. We will need only its rest-frame version. It follows from the following expansion of the rest-frame components of the electric induction field in the vicinity of the particle:

$$
D^{k}=\frac{e}{4 \pi}\left[\frac{x^{k}}{r^{3}}-\frac{1}{2 r}\left(a_{i} \frac{x^{i} x^{k}}{r^{2}}+a^{k}\right)\right]+O(1) .
$$

Here $a=\left(a^{k}\right)$ is the acceleration of the particle (in the rest frame we have $a^{0}=0$ ) and $O(1)$ denotes the nonsingular part of the field. The magnetic field $B^{k}(r)$ does not contain any singular part.

We stress that the relation between the particle's acceleration and the $r^{-1}$ component of $D$, implied by formula (9), is not a new element of the theory. Although the author has never seen it written in this explicit form, it is implicitly contained in the formula for Lienard-Wiechert potentials, (e.g. formula (14.14) in Jackson's book [10] or formula (6-62) in Rohrlich's book [2]). Choosing the rest frame $(v=0)$ and calculating the limit of the retarded (or advanced) field in the neighbourhood of the particle one easily proves Eq. (11) for the retarded (advanced) solution. Any regular solution of (1) differs from the retarded (advanced) 
solution by a smooth solution of the free Maxwell equation, i.e. the one which does not change the singular part of (9). Hence, the formula is valid universally (in Ref. [6] we give an independent proof, which does not make use of any specific - retarded or advanced - solutions).

It is useful to rewrite Eq. (9) as a condition for the behaviour of the radial component $D^{r}$ of $\boldsymbol{D}$. For this purpose we expand $D^{r}$ in the neighbourhood of the particle into powers of $r$

$$
D^{r}(r)=\frac{1}{4 \pi}\left(\frac{e}{r^{2}}+\frac{\alpha}{r}\right)+\beta+O(r)
$$

where by $O(r)$ we denote terms vanishing for $r \rightarrow 0$ like $r$ or faster. For a given value of $r$ both sides of Eq. (10) are functions of the angles (only the $r^{-2}$ term is angle-independent).

It is easily seen that the condition (9) is equivalent to the following constraint:

$$
\alpha=-e a_{i} \frac{x^{i}}{r} \text {. }
$$

IIere we use the one-to-one correspondence between the 3-vectors and the "dipole-like" functions of angles, i.e. functions of the form $\left(a_{i} x^{i}\right) / r$. Formula (11) means; in particular, that the quadrupole and higher harmonics of the function $\alpha$ do vanish.

The above observation suggests the following point of view. Maxwell equations (1) have to be solved as a boundary problem rather than as a distribution equation on the entire space-time $\mathcal{M}$. More precisely, we consider the evolution of the free field in the region $\mathcal{M}_{\zeta}:=\mathcal{M}-\{\zeta\}$, that means outside of the trajectory. Equation (11) provides the boundary condition on the boundary $\partial \mathcal{M}_{\zeta}$ of $\mathcal{M}_{\zeta}$.

There are various ways to set up the boundary value problem in electrodynamics. The best known are Dirichlet and Neumann problems for electrodynamical potentials. Here we found it most useful to assume boundary conditions corresponding to the given double layers of electric and magnetic dipoles. In this way the values $D^{\perp}$ and $B^{\perp}$ (components of both the electric and the magnetic fields, normal to the boundary) are controlled. It was shown in Ref. [11] that under this choice of boundary conditions on $\partial V$, the IIamiltonian generating the evolution of the field in the region $V$ coincides with the total energy of the field.

The particle trajectory may be considered as a limit of tiny world-tubes. It may be shown that the natural limit of the boundary conditions for $D^{\perp}$ and $B^{\perp}$ consists in fixing their singular parts at zero. Thus, for an a priori given particle trajectory, Eq. (11) provides the condition for $D^{\perp}$ on its boundary. The corresponding condition for the magnetic field follows from the assumption about the vanishing magnetic moment of the particle: $B^{r}(r)=O(1)$ (i.e. the singular part of $B^{r}$ vanishes). The reader may easily check that, for a given trajectory, the homogeneous Maxwell equations outside of the particles together with the above boundary conditions are equivalent to the non-homogeneous system (1).

Now, let us consider an extended system, where also the particle trajectory is treated as a dynamical object and Eq. (11) is one of dynamical equations, enabling to calculate the particle's acceleration from the initial data. Observe, that a remarkable phenomenon occurs. Despite the fact that the time derivatives 
$(\dot{\boldsymbol{D}}, \dot{\boldsymbol{B}}, \dot{\boldsymbol{q}}, \dot{\boldsymbol{v}})$ of the Cauchy data $(\boldsymbol{D}, \boldsymbol{B}, \boldsymbol{q}, \boldsymbol{v})$ are uniquely determined by the data themselves, the evolution is not uniquely determined. This is due to the fact that, after upgrading Eq. (11) to the level of dynamical equations, we can no longer use it as a boundary condition. Hence, Maxwell theory alone provides sufficiently many dynamical equations of the theory, but a new boundary condition is necessary. The new element of the theory is precisely this missing boundary condition, implied by the conservation law for the renormalized total four-momentum, defined in the previous section.

The situation is similar to that in classical theory of a finite elastic body $V$ (e.g. a finite piece of an elastic string). The theory contains sufficiently many dynamical equations, but the evolution is not uniquely determined unless we restrict ourselves to the class of solutions fulfilling the appropriate boundary conditions on the boundary $\partial V$.

\section{Fundamental equation. Correspondence principle}

Here we present the fundamental equation of our theory, which is the necessary and sufficient condition for the total momentum conservation (7). It is formulated as a boundary condition for the behaviour of the field in the vicinity of the particle. Again, for the sake of simplicity, we write it down in the particle co-moving frame, i.e. in the form of a relation between differcnt terms of expansion (10). The relation reads

$$
\mathrm{DP}\left(m \alpha+e^{2} \beta\right)=0,
$$

where by $\operatorname{DP}(f)$ we denote the dipole part of the function $f$ on the sphere $S^{2}$. This equation (together with the vanishing of the singular part of the magnetic field $B$ ) provides the missing boundary condition for the behaviour of the field $D^{r}$ on the boundary $\partial \mathcal{M}_{\zeta}$ of the region $\mathcal{M}_{\zeta}$. Dynamical equations of the theory are, therefore, the Maxwell equations alone. They will determine uniquely the evolution of the system composed of particles and fields if we restrict ourselves to solutions fulfilling the boundary condition (12). In this way the system becomes an infinite-dimensional dynamical system (it is even a Hamiltonian system).

Although the Lorentz force acting on particles is always ill defined, it is contained in our theory as a limiting case, when particles are very light and carry a very small charge. Indeed, passing to the limit with $m \rightarrow 0$ and $e \rightarrow 0$ (but keeping their ratio constant), we obtain the standard theory of test particles, where the field is not influenced by the particles and the particles are influenced by fields according to the Lorentz force. To prove this feature of our theory we use Eq. (11) to rewrite Eq. (12) in the following, equivalent form:

$$
\operatorname{DP}(\beta)=\frac{m}{e} a_{i} \frac{x^{i}}{r}
$$

For $e=0 \mathrm{Eq}$. (1) reduces to the free Maxwell equation and the first two terms of Eq. (10) vanish. The field is, therefore, regular at $r=0$. For regular fields the radial component of $\boldsymbol{D}$ contains only the dipole part: $\mathrm{DP}(\beta)=\left(D_{i}(0) x^{i}\right) / r=\left(E_{i}(0) x^{i}\right) / r$ (higher harmonics behave like $O(r)$ and the electric field $\boldsymbol{E}$ coincides with the 
electric induction field). Therefore, Eq. (13) is equivalent to the equation defining the Lorentz force in the rest frame

$$
E^{i}(0)=\frac{m}{e} a^{i} \text {. }
$$

Hence, we have proved that our theory fulfills the correspondence principle with the Lorentz theory of test particles. We stress, however, that the interpretation of Eq. (13) as the Lorentz force is not possible for a particle with finite charge and mass. Due to the first two (singular) terms in Eq. (9), any attempt to define the Lorentz force in this case leads to infinite expressions. Considering the particle as a limit of a small, spherical droplet of matter, the $r^{-2}$ term can be eliminated (the forces, although big, cancel out because of the spherical symmetry). However, such a "renormalization" fails for the $r^{-1}$ term which will always be infinite. We see that the Lorentz force cannot be meaningfully defined for point particles not only because of the "proper field", which increases like $r^{-2}$, but also because of the infinite contribution of the remaining "radiation field". Moreover, also the non-singular term of $\boldsymbol{D}$ cannot be used to define the Lorentz force in a reasonable way, because its value at $r=0$ cannot be uniquely defined (different values of its limit at $r=0$ may be obtained if we approach the origin from different directions - see formulae (34), (35) and the discussion which follows).

We conclude that the Lorentz force is a well-defined concept for test particles only and has no meaning for a finite particle. Nevertheless, the dynamics of the system composed of particles and fields, given by Maxwell equations and the boundary condition (12), is well defined and fully deterministic: initial data for particles and fields uniquely determine the entire history of the system.

Thus, we have shown that the evolution of interacting particles and fields is a problem with moving boundary for the Maxwell equations. The particle trajectory plays the role of the boundary. Such problems are often considered in the theory of continuous media (see [13]) and this analogy is a good guiding principle in the analysis of our problem.

\section{Co-moving description of the field dynamics. Idea of the proof of the fundamental equation}

The easiest way to prove the equivalence of Eq. (12) with the momentum conservation (7) consists in rewriting Maxwell equations in the particle's rest frame. This is not an inertial frame, but an accelerated one, described by a curvilinear system of space-time coordinates.

For this purpose let us parameterize the trajectory $\zeta$ by its proper time $\tau$. At each point $\left(y^{\mu}(\tau)\right) \in \zeta$ of the trajectory take the 3-dimensional hyperplane $\Sigma_{\tau}$ orthogonal to $\zeta$. We shall call $\Sigma_{\tau}$ the "rest frame surface". Choose on $\Sigma_{\tau}$ any system $\left(x^{i}\right)$ of cartesian coordinates, such that the particle is located at its origin (i.e. at the point $x^{i}=0$ ).

Let us identify the space-time with the disjoint sum of rest frame surfaces $\Sigma_{\tau}$, each of them corresponding to a specific value of the coordinate $x^{0}:=\tau$ and parameterized by the coordinates $\left(x^{i}\right)$. This way we obtain a system $\left(x^{\alpha}\right)=$ $\left(x^{0}, x^{k}\right)$ of "co-moving" coordinates in a neighbourhood of $\zeta$. Unfortunately, it is 
not a global system because different $\Sigma$ 's may intersect. Nevertheless, we will use it globally to describe the evolution of the electromagnetic field from one $\Sigma_{\tau}$ to another. Because of the hyperbolic structure of the theory, this is a well-defined problem. Formally, we will simply rewrite the field equations with respect to the curvilinear system $\boldsymbol{x}^{\boldsymbol{\alpha}}$.

For this purpose we need to find the space-time metric components. Because $\left(x^{k}\right)$ are cartesian coordinates on $\Sigma$, the space-space components of $g$ are trivial: $g_{i j}=\delta_{i j}$. It is easy to see that the only non-trivial components of $g$ are: the lapse function

$$
N=\frac{1}{\sqrt{-g^{00}}}=1+a_{i} x^{i}
$$

where $a=\left(a^{i}\right)$ is the particle's acceleration vector in the co-moving frame, and the (purely rotational) shift vector

$$
N_{m}=g_{0 m}=\epsilon_{m k l} \omega^{k} x^{l},
$$

where the rotation vector $\omega$ depends upon the coordination of the cartesian systems $\left(x^{i}\right)$ between different $\Sigma$ 's. The simplest $O(3)$-coordination consists in Fermi-propagating each of the $x^{k}$-axis along $\zeta$. In this case the shift simply vanishes and we have $g_{0 m}=0$.

The components of the flat, Minkowski connection, can be obtained as Christoffel symbols for the above metric

$$
\Gamma_{00}^{0}=\frac{\dot{a}_{j} x^{j}}{1+a_{i} x^{i}}, \quad \Gamma_{0 k}^{0}=\frac{a_{k}}{1+a_{i} x^{i}}, \quad \Gamma_{00}^{k}=\left(1+a_{i} x^{i}\right) a^{k},
$$

and the remaining components vanish.

The metric and the connection are degenerate at singular points of the identification map (i.e. where the identification is locally non-invertible because adjacent $\Sigma$ 's intersect, i.e. where $N=0$ ). Nevertheless, electrodynamics may be rewritten in coordinates $\left(x^{\alpha}\right)$ just as in any other curvilinear system of coordinates, using the above representation of the space-time geometry.

In particular, formula (6) rewritten in curvilinear coordinates, gives the following expressions for the rest-frame components of the total four-momentum:

$$
\begin{aligned}
& \mathcal{H}=-\mathcal{P}_{0}=m-\int_{\Sigma}\left(T_{0}^{0}-T_{0}^{0}\right) \mathrm{d}^{3} x \\
& \mathcal{P}_{k}=\int_{\Sigma}\left(1+a_{i} x^{i}\right) T^{0}{ }_{k} \mathrm{~d}^{3} x
\end{aligned}
$$

where $T^{0}{ }_{0}=e^{2} / 32 \pi^{2} r^{4}$ is the energy density of the Coulomb field

$$
D_{0}=\frac{e r}{4 \pi r^{3}} \text {. }
$$

The factor $\left(1+a_{i} x^{i}\right)$ in formula (19) is necessary because only the co-vector $\left(1+a_{i} x^{i}\right) \mathrm{d} x^{0}$ (and not $\left.\mathrm{d} x^{0}\right)$ is constant on $\Sigma$ (similar corrections in Eq. (18), corresponding to the upper zero and to the lower zero, cancel each other). 
To calculate the time derivatives of $\mathcal{P}_{\alpha}$ we use the conservation laws

$$
\begin{aligned}
0= & \nabla_{\alpha} T_{0}^{\alpha}=\partial_{\alpha} T_{0}^{\alpha}+T^{\beta}{ }_{0} \Gamma_{\beta \alpha}^{\alpha}-T^{\alpha}{ }_{\beta} \Gamma_{0 \alpha}^{\beta} \\
& =\partial_{\alpha} T^{\alpha}{ }_{0}-T^{0}{ }_{k} a^{k}\left(1+a_{i} x^{i}\right)
\end{aligned}
$$

and

$$
\begin{aligned}
0= & \nabla_{\alpha} T^{\alpha}{ }_{k}=\partial_{\alpha} T_{k}^{\alpha}+T_{k}^{\beta} \Gamma_{\beta \alpha}^{\alpha}-T^{\alpha}{ }_{\beta} \Gamma_{k \alpha}^{\beta} \\
& =\partial_{\alpha} T^{\alpha}{ }_{k}+T^{0}{ }_{k} \frac{\dot{a}_{j} x^{j}}{1+a_{i} x^{i}}+T^{l}{ }_{k} \frac{a_{l}}{1+a_{i} x^{i}}-T^{0}{ }_{0} \frac{a_{k}}{1+a_{i} x^{i}}
\end{aligned}
$$

implied by the Maxwell equations. The last equation is equivalent to

$$
\partial_{\alpha}\left(\left(1+a_{i} x^{i}\right) T_{k}^{\alpha}\right)=T^{0}{ }_{0} a_{k} .
$$

Applying Eq. (21) to Eq. (18) we obtain

$$
\begin{aligned}
\dot{\mathcal{H}}= & -\int_{\Sigma} \partial_{0} T^{0}{ }_{0} \mathrm{~d}^{3} x \\
& =-\lim _{r_{0} \rightarrow 0} \int_{\left\{r=r_{0}\right\}} T^{\perp}{ }_{0} \mathrm{~d} \sigma-\int_{\Sigma} T^{0}{ }_{k} a^{k}\left(1+a_{i} x^{i}\right) \mathrm{d}^{3} x=-a_{k} \mathcal{P}^{k}
\end{aligned}
$$

because the surface integral vanishes at the limit $r_{0} \rightarrow 0$, as may be easily shown.

Applying Eq. (23) to Eq. (19) we obtain

$$
\begin{aligned}
\dot{\mathcal{P}}_{k} & =-\int_{\Sigma} \partial_{0}\left(\left(1+a_{i} x^{i}\right) T^{0}{ }_{k}\right) \mathrm{d}^{3} x \\
& =-\lim _{r_{0} \rightarrow 0}\left\{\int_{\left\{r=r_{0}\right\}}\left(1+a_{i} x^{i}\right) T^{\perp} \mathrm{d} \sigma-a_{k} \int_{\left\{r>r_{0}\right\}} T^{0}{ }_{0} \mathrm{~d}^{3} x\right\} \\
& =-\lim _{r_{0} \rightarrow 0}\left\{\frac{e^{2}}{8 \pi r_{0}} a_{k}+\int_{\left\{r=r_{0}\right\}}\left(1+a_{i} x^{i}\right) T^{\perp}{ }_{k} \mathrm{~d} \sigma\right. \\
& \left.-a_{k} \int_{\left\{r>r_{0}\right\}}\left(T^{0}{ }_{0}-T^{0}{ }_{0}\right) \mathrm{d}^{3} x\right\} .
\end{aligned}
$$

In this case the surface integral does not vanish. Using formula (9) to calculate the contribution of singular terms of the field, we may easily check that it is composed of two terms: (1) a $\left(r^{-1}\right)$-term which annihilates the corresponding term coming from the Coulomb field, and (2) the regular term equal to the $k$-th component $\beta_{k}$ of the dipole part of the function $\beta$. The component is defined by the decomposition

$$
\operatorname{DP}(\beta):=\beta_{k} \frac{x^{k}}{r} \text {. }
$$

Finally, we obtain the formula

$$
\dot{\mathcal{P}}_{k}=-e \beta_{k}-a_{k}(\mathcal{H}-m)=-a_{k} \mathcal{H}+\left(m a_{k}-e \beta_{k}\right) .
$$

On the other hand, the four-momentum conservation law written in the co-moving reference frame reads

$$
\nabla_{0} \mathcal{P}^{\alpha}=\partial_{0} \mathcal{P}^{\alpha}+\Gamma_{0 \beta}^{\alpha} \mathcal{P}^{\beta}=0,
$$


where $\left(\mathcal{P}^{\alpha}\right)=\left(\mathcal{H}, \mathcal{P}^{k}\right)$ and the value of the connection components has to be taken on the trajectory (i.e. $\Gamma_{0 k}^{0}=a_{k}$ and $\Gamma_{00}^{k}=a^{k}$ ). Hence, the system (7) and (8) is equivalent to the following system of equations for the rest-frame components of the four-momentum:

$$
\dot{\mathcal{H}}=-a_{k} \mathcal{P}^{k}
$$

and

$$
\dot{\mathcal{p}}^{k}=-\boldsymbol{a}^{k} \mathcal{H}
$$

Comparing them with Eqs. (24) and (27) we conclude that one among them is always satisfied. We see that the necessary and sufficient condition for the total four-momentum conservation is given by equation

$$
m a_{k}-e \beta_{k}=0 .
$$

equivalent to the fundamental Eq. (12).

\section{Many particles. Interaction with heavy external devices}

Although up to now we have considered only a single particle interacting with the Maxwell field, our theory is formulated in terms of local quantities and can be immediately extended to the case of many particles: the field outside the particles has to fulfill the Maxwell equations with the total current being the sum of the contributions from all the trajectories, and the boundary condition (12) has to be satisfied on each trajectory separately.

The above law can be derived from a renormalized four-momentum formula analogous to (6), in which there is a term $\boldsymbol{T}_{(t)}{ }_{\lambda}$ and a term $m u_{\lambda}$ for each particle separately. Any variation of $\Sigma$ which does not change the intersection points with all the particle trajectories, does not change also the value of $p_{\lambda}$. To prove this fact we use the same arguments as in the case of one particle. Hence, the total four-momentum defined this way depends upon the choice of the intersection points only: one for cach trajectory. Assuming the independence of $p_{\lambda}$ upon the intersection points one finally gets the fundamental equation separately on each trajectory.

Consider now the limiting case of the two particles problem, when one of the two particles is very heavy and practically does not move: its acceleration $a$ calculated from Eq. (11) is of the order of $1 / m$ which, for sufficiently large $m$, can be neglected. This means that we do not need to satisfy the boundary condition for the heavy particle (its "infinitesimally small" acceleration fits any value of $\operatorname{DP}(\beta)$ in formula (13) when multiplied by its "infinitely big" mass). The boundary condition has to be satisfied for the light particle only and our co-moving description of the field may be used also in this case.

The heavy particle may be replaced by any heavy (macroscopic) device containing charged bodies, magnets etc. (e.g. an accelerator). Let $\left(\phi^{\text {ext }}, f^{\text {ext }}\right)$ be an exact solution of our "super theory" of interacting matter fields and electromagnetic field, which represents such a device and its own electromagnetic field. We call the field "external", because it plays such a role for our (relatively light) particle interacting with the device. 
Suppose now that there is another solution ( $\left.\phi^{\text {total }}, f^{\text {total }}\right)$ of the "super theor $\eta^{\prime}$ which represents such an interaction. We assume that the total strong field region of the new solution consists of the device's own strong field region (i.e. the strong field region of the previous solution) and the separate region concentrated around the approximate trajectory $\zeta$ of the light particle. It is important to assume that the trajectory does not intersect the strong field region of the device.

Let us define the "radiation field" $f$ as a difference between the two solutions

$$
f_{\mu \nu}^{\text {total }}=f_{\mu \nu}^{\text {ext }}+f_{\mu \nu} .
$$

The radiation field satisfies the Maxwell equations outside of the matter, because both $f_{\mu \nu}^{\text {total }}$ and $f_{\mu \nu}^{\text {ext }}$ do. The boundary condition (12) for $f^{\text {total }}$ on the trajectory $\zeta$ can be rewritten in terms of the boundary condition for the radiation field

$$
\mathrm{DP}\left(m \alpha+e^{2} \beta\right)=-e^{2} D_{i}^{\mathrm{ext}} \frac{x^{i}}{r}
$$

where $D_{i}^{\text {ext }}$ are the electric induction components of the field $f^{\text {ext }}$, corresponding to the actual position of the particle and calculated in the particle rest frame. This is due to the fact that $f^{\text {ext }}$ is a smooth field in the neighbourhood of the particle trajectory. Therefore, $\alpha^{\text {total }}=\alpha$ and $\beta^{\text {total }}=\beta+\left(D_{i}^{\text {ext }} x^{i}\right) / r$, where $\alpha$ and $\beta$ denote the corresponding quantities for the radiation field $f$.

We see that the dynamical equations for the field $f$ are the same as in the one-particle problem. The influence of the external field is manifested in the non-homogeneous boundary condition (33), which now replaces the homogeneous condition (12).

The right hand side of (33) can be written explicitly in terms of the laboratory-frame components $\mathcal{E}^{i}(t, q)$ and $B^{i}(t, q)$ of the external field if we know explicitly the Poincaré transformation relating the laboratory frame with the rest frame.

\section{Radiative friction as a result of "fine tuning" of the field}

In the present section we will describe a particular class of exact solutions of our theory. Each solution of this class can be obtained by solving a specific ordinary differential equation for the trajectory, with the additional term containing third-order derivatives of the particle position. There is a one-parameter class of such "radiative" terms and Dirac's term proposed in Ref. [2] corresponds to a specific choice of this parameter. Another possibility could be e.g. an "anti-Dirac" term or any convex combination of these two. Some of the solutions obtained this way realize Dirac's idea of "radiative friction". Our theory is symmetric with respect to time reversal and, therefore, there are also "self-accelerating" solutions obtained this way. Also solutions without any friction or self-acceleration can be obtained via a similar method.

The physical importance of all these solutions is very limited, because each of them corresponds to a very specific choice of initial data for the field. To maintain specific, purely mechanical behaviour of the particle (e.g. friction or self-acceleration) the field on the entire Cauchy surface $\Sigma$ has to be chosen in a way perfectly tuned to the prescribed shape of the particle trajectory. In most 
cases, such a "fine tuning" needs an infinite amount of "infra-red" field energy, since the field initial data do not fall off sufficiently fast at infinity. Generic initial data do not satisfy this "fine tuning" condition and, therefore, do not admit this method of solution.

There are, however, physical situations in which solutions obtained via Dirac's method are physically meaningful. Such situations correspond to trajectories which are asymptotically free for $t \rightarrow-\infty$. If the external field $f^{\text {ext }}$ admits such a trajectory for a test particle, then there is a preferable choice of the initial data for the radiation field $f$ at $t \rightarrow-\infty$, namely the boosted Coulomb field surrounding the uniformly moving particle. As we shall see in the sequel, the exact solution of our theory with such initial data can be obtained by solving the Lorentz-Dirac equation for the trajectory and taking as the radiation field $f$ the retarded Lienard-Wiechert solution corresponding to such a trajectory. This method fails in case of closed trajectories (e.g. for the Kepler problem), when there is no natural decomposition of the electromagnetic field into "incoming" and "retarded" fields. Here, the choice of the time-arrow (e.g. friction or self-acceleration) corresponds to an arbitrary choice of the fine-tuned, infra-red-divergent initial data. Meaningful physical situations correspond to a local, non-linear interaction of particles and fields and cannot be classified either as an example of the radiative friction or of the radiative acceleration of the particles. To obtain them we need to solve our system of genuine partial differential equations, which does not reduce to any system of ordinary differential equations.

The method we are going to describe is based on the observation that for the retarded field $D_{\text {ret }}$ the expansion (10) assumes the following form:

$$
D_{\mathrm{ret}}^{r}=\frac{e}{4 \pi}\left\{\frac{1}{r^{2}}-\frac{a_{i} x^{i}}{r^{2}}+\frac{3}{8}\left[3\left(\frac{a_{i} x^{i}}{r}\right)^{2}-a^{2}\right]+\frac{2 \dot{a}_{i} x^{i}}{3 r}\right\}+O(r),
$$

(this formula can be easily obtained from the formula (6-62) in Rohrlich's book [2]). A similar formula for the advanced field differs only by the sign of the last term representing the dipole part of $\beta$

$$
D_{\mathrm{adv}}^{r}=\frac{e}{4 \pi}\left\{\frac{1}{r^{2}}-\frac{a_{i} x^{i}}{r^{2}}+\frac{3}{8}\left[3\left(\frac{a_{i} x^{i}}{r}\right)^{2}-a^{2}\right]-\frac{2 \dot{a}_{i} x^{i}}{3 r}\right\}+O(r) .
$$

The last two terms in both formulae give the total value of $\beta$. The first of the two represents the non-vanishing quadrupole part of $\beta$ (its existence proves that the value $\widetilde{D}(0)$ of the non-singular part cannot be consistently defined). Equation (33) implies that the retarded field satisfies the equations of our theory if and only if the trajectory satisfies the following third-order ordinary differential equation:

$$
m a_{i}=e D_{i}^{\text {ext }}+\frac{e^{2}}{6 \pi} \dot{a}_{i}
$$

The above equation has been written with respect to the particle rest frame and in terms of the 3-dimensional objects $a$ and $\dot{a}$. It is obviously equivalent to the Lorentz-Dirac equation written in the 4-dimensional notation

$$
m a^{\mu}=e F_{\mathrm{ext}}^{\mu \nu} v_{\nu}+\frac{e^{2}}{6 \pi}\left(\dot{a}^{\mu}-a^{\nu} a_{\nu} v^{\mu}\right)
$$


(with respect to the corresponding formula in Rohrlich's book there is an additional factor $4 \pi$ due to the fact that we use Heaviside-Lorentz system of units). Finding a trajectory, which satisfies this equation and calculating the corresponding retarded field we find an exact solution of our theory. Similarly, we may use advanced fields. Due to equation (35) the corresponding equation for the trajectory will differ from the above one by the sign of the last term.

A more general "Ansatz" is a combination of advanced and retarded solutions

$$
f:=\frac{1+\lambda}{2} f_{\text {ret }}+\frac{1-\lambda}{2} f_{\text {adv }}
$$

where $\lambda$ is a real parameter. The field provides an exact solution of our theory if and only if the trajectory satisfies the "Lorentz-Dirac type" equation

$$
m a^{\mu}=e F_{\text {ext }}^{\mu \nu} v_{\nu}+\lambda \frac{e^{2}}{6 \pi}\left(\dot{a}^{\mu}-a^{\nu} a_{\nu} v^{\mu}\right) .
$$

Dirac's case $\lambda=1$ corresponds to the retarded fields, the "anti-Dirac" case $\lambda=-1$ - to advanced fields. The specific case $\lambda=0$ corresponds to the pure Lorentz equation with external forces

$$
m a^{\mu}=e F_{\mathrm{ext}}^{\mu \nu} v_{\nu} \text {. }
$$

The above method is useless if the trajectory is bounded, because then the retarded and advanced fields behave like $1 / r$ for $r \rightarrow \infty$ and the field energy, necessary to maintain such a behaviour of the particle, is infinite.

We stress that the Lorentz-Dirac equation describes only one possible behaviour of the particle interacting with the field, corresponding to a specific choice of the initial data. We conclude that it does not describe a fundamental law of nature but only a special family of solutions of the complete theory described in the present paper.

We stress also that there is even no universal choice of the sign of the parameter $\lambda$ in Eq. (39), which would always correspond to friction rather than to self-acceleration. The reader may easily check that for the free particle (i.e. $f_{\text {ext }}=0$ ) positive $\lambda$ leads to self-acceleration, whereas for the Kepler problem they lead to radiative friction. Of course, the modulus of $\lambda$ is also arbitrary $(\lambda=5$ is as legitimate as Dirac's $\lambda=1$ ).

But the main drawback of the Lorentz-Dirac theory consists in the contradiction between the fundamental role of the Lorentz force and the fact that there is no way to define it consistently. This paradox may be avoided only if one treats the interaction between particles and fields as a problem with moving boundary. IIere, the notion of the Lorentz force is meaningful for test particles only.

\section{Acknowledgments}

I am very much indebted to Professor Iwo Bialynicki-Birula for infinitely many discussions which influenced very much my understanding of the physical meaning of very complicated mathematical structures, describing the theory in its primary version. Professor Białynicki also read carefully the successive versions of the present paper and suggested to me a lot of important improvements in its final formulation. Many thanks are due to Professor Joshua Goldberg, who "forced" me to find a simple proof (presented in Sec. 5 of this paper) of the fundamental equation. 


\section{References}

[1] J.A. Wheeler, R.P. Feynmann, Rev. Mod. Phys. 21, 425 (1949).

[2] P.A.M. Dirac, Proc. R. Soc. A 167, 148 (1938); R. Haag, Naturforsch. A 10, 752 (1955); F. Rohrlich, Classical Charged Particles, Addison-Wesley, Reading 1965.

[3] W. Pauli, M. Fierz, Nuovo Cimento 15, 167 (1938) or W. Pauli, in: Collected Scientific Papers, Eds. R. Kronig, V.F. Weisskopf, Wiley, New York 1964.

[4] L. Galgani, C. Angaroni, L. Forti, A. Giorgilli, F. Guerra, Phys. Lett. A 139, 221 (1989); G. Arioli, L. Galgani, ibidem 162, 313 (1991).

[5] M. Born, L. Infeld, Proc. R. Soc. A 144, 425 (1934); E. Feenberg, Phys. Rev. 47, 148 (1935).

[6] J. Kijowski, General Relativity and Gravitation Journal, in print.

[7] J. Kijowski, W.M. Tulczyjew, A Symplectic Framework for Field Theories, Lecture Notes in Physics, Vol. 107, Springer-Verlag, Berlin 1979.

[8] L. Landau, E. Lifshitz, The Classical Theory of Fields, Pergamon, New York 1962.

[9] J. Kijowski, in: Proc. of Journées Relalivistes 1983, Torino, Eds. S. Benenti, M. Francaviglia, D. Galetto, Pitagora Editrice, Bologna 1985, p. 205; J. Kijowski, in: Gravitation, Geometry and Relativistic Physics, Ed. Laboratoire "Gravitation et Cosmologie Relativistes" Université P. et M. Curie et C.N.R.S., Institut Henri Poincaré (Paris), Lecture Notes in Physics, Vol. 212, Springer-Verlag, Berlin 1984, p. 40; J. Jezierski, J. Kijowski, in: Proc. of the Sixth Marcell Grossmann Meeting on General Relativity, Part A, Eds. H. Sato, T. Nakamura, World Sci., Singapore 1992, p. 123.

[10] J.D. Jackson, Classical Electrodynamics, 2-nd ed., Wiley, New York 1975.

[11] J. Jezierski, J. Kijowski, Gen. Relativ. Grav. 22, 1283 (1990).

[12] I. Białynicki-Birula, Z. Białynicka-Birula, Quantum Electrodynamics, Pergamon, Oxford 1975.

[13] A. Friedman, Variational Principles and Free Boundary Problems, Wiley, New York 1982. 